\title{
Plant based phytochemicals less targeted for the COVID-19 treatments: some observations
}

\section{Opinion}

Severe Acute Respiratory Syndrome and immunity lost healthcare systems due to SARS-CoV-2 ended in to a disaster at global scale. Search for complementary and alternative medicinal systems becomes the need of the hour. Native communities residing in remote rural areas practiced plant-based remedies as curative and preventive against COVID-19 outbreaks. Aboriginal people are the real hub of traditional knowledge. The COVID-19 pandemic, which particularly affects people above sixty, reflects the immunity issues connected with the disorder. It is therefore important to explore ethnobotanical interdisciplinary research towards shared benefits with local indigenous knowledge against such pandemic diseases.

Holistic approach with personalized treatment and syndrome differentiation forms the base of traditional Chinese medicine. Indian system such as Ayurveda, Unnani and Homeopathy were practiced by experienced people based on certain scientific reasons in eradicating and defending such multi resistant pathogens. The above statements may be substantiated by few examples. Turmeric is a documented proven indigenous system against diverse respiratory issues such as asthma, bronchial hyperactivity, allergy, liver disorders, anorexia, rheumatism, diabetic wounds, runny nose, cough, and sinusitis. Similarly, garlic another traditional bioresource accounts rich sulfur containing amino acids and other active molecules seem to initiate and induce immune system by making macrophages or killer cells more active. Tulsi, another traditional drug boost defence against infectious agents by triggering immune responses in non-stressed and stressed human and animal systems.

Cinnamon, the most common spices and flavoring additive and function as neuro, hepato, cardio and gastroprotective roles via antioxidant and anti-inflammatory properties Black pepper extracts is used for treating cold and cough. It also shows relief from sinusitis and nasal congestion. i.e., the expectorant property helps to break up the mucus and phlegm accumulations in the respiratory tract. This mode of natural irritant quality helps to expel unwanted loosened materials through the act of sneezing or coughing, which expels the material from the body and helps to recover from infection or illness that caused the deposition in the affected areas. Therefore, it is ideal agaisnt respiratory disorders and acute inflammatory disorders.

Ginger, another traditional curative and can be used for throat infections and to relieve congestion in sinusitis. It reduces fever in colds and flu and suppresses the dry, irritating cough in laryngitis by increasing human bronchial smooth muscle cell migration and proliferation and reversing phthalate ester-mediated airway remodeling. It has been found effective against the flu virus, due to its warm and bitter property. Many sesquiterpenes, like

\author{
Volume 14 Issue 3 - 202 I
}

K Murugan,' Remya Krishnan ${ }^{2}$

'Department of Botany, CISSA - Centre for Innovation in

Science and Social Action, India

${ }^{2}$ Department of Botany, NSS College, Cherthala, Kerala, India

Correspondence: K Murugan, Department of Botany, CISSA Centre for Innovation in Science and Social Action, India, Email harimurukan@gmail.com

Received: January II, 202I | Published: May 28, 2021

$\beta$-sesquiphellandrene, has been shown to have proven anti-rhinoviral activity under in vitro trials. ${ }^{1}$ Pandey et al., ${ }^{2}$ documented that emetine, an alkaloid extracted from the root of Psychotria ipecacuanha (ipecac root), that was determined to be a broad-spectrum inhibitor regulating different CoVs under in vitro trials and was found to inhibit SARS$\mathrm{CoV}-2$ replication. Interestingly, many lead molecules are isolated and capitalized by pharmacology related industries. Thus, there is ample scope for the scientific community to competently tap the traditional knowledge and take active scientific research up to the clinical trials so that potential anti-COVID bio-drugs can be designed and thereby saving human life from pandemic diseases.

\section{Acknowledgments}

None.

\section{Conflicts of interest}

Author declares that there are no conflicts of interest.

\section{Funding}

None.

\section{References}

1. Agarwal P, Goyal A. Combing through traditional texts to prevent covid-19 - a scientific approach. International Journal of Pharmaceutical Sciences and Research. 2021;12(1):8-21.

2. Pandey A, Khan M, Hamurcu M, et al. Natural Plant Products: A Less Focused Aspect for the COVID-19 Viral Outbreakront. Front Plant Sci. 2020;11:568890. 